\title{
Blind Equalization in OFDM Systems by Channel Shortening and Channel Diversity Exploitation
}

\author{
Cang Yan \\ College of Information and \\ Communication Engineering \\ Harbin Engineering University, \\ No 145 , Nantong Str. Nan \\ Gang Dist. Harbin 15000121 - \\ A Building. Communication \\ and Circuit Lab, PRC
}

\author{
Emmanuel A Mwangosi \\ College of Information and \\ Communication Engineering \\ Harbin Engineering University, \\ No 145, Nantong Str. Nan \\ Gang Dist. Harbin 15000121 - \\ A Building. Communication \\ and Circuit Lab, PRC
}

\author{
Naveed Ur Rehman \\ College of Information and \\ Communication Engineering \\ Harbin Engineering University, \\ No 145 , Nantong Str. Nan \\ Gang Dist. Harbin 150001 21- \\ A Building. Communication \\ and Circuit Lab, PRC
}

\begin{abstract}
We propose two alternative approaches for blind channel shortening to design time domain finite impulse response equalizer (TDE) in multicarrier single input, multiple output systems. The first approach we utilize the constant envelop of the transmitted signal called constant modulus (CM). Second approach is based on decision directed algorithm, where we update time domain equalizer using decision directed cost function. These cost function have been widely studied for single carrier systems, implementing them in multicarrier scenario is of great importance. Simulations shows they outperform classical multicarrier equalization by restoration of redundancy (MERRY) and Carrier nulling algorithm (CNA) that utilizes redundancies inherent in carriers in terms of bit error rate.
\end{abstract}

\section{General Terms}

Channel equalization algorithms in wireless communication.

\section{Keywords}

Blind equalization, Constant modulus algorithm, decision directed algorithm, SIMO, OFDM.

\section{INTRODUCTION}

Future communication systems aim to provide high data rate with reliability. The main challenge to achieve this goal is the dispersive effects of communication channels, which leads to inter-symbol interference (ISI). Multicarrier modulation techniques such as OFDM and DMT have been widely used in high speed digital communication systems to combat channel frequency selectivity, the technique work effectively when the delay spread of the channel does not exceed the cyclic prefix $(\mathrm{CP})$ inserted at the transmitter [1]. Extending the CP length is bandwidth inefficiency while insufficient CP length results in inter-carrier (ICI) and inter-symbol (ISI) interference due to subcarriers orthogonality destruction. In this case, equalization at the receiver to shorten the effective channel to appropriate channel becomes important. Channel shortening is done by means of a time domain equalizer (TDE), which is finite impulse response filter (FIR). Cascaded channel-equalizer arrangement produces the effective shortened channel impulse response. Most of channel shortening schemes utilizes training sequences which is bandwidth inefficient [2]. These methods have been studied in the context of wire line systems such as Digital subscriber line (xDSL) [2]. High complexity nature and being non-adaptive have made more quests for better method [3]. Blind adaptive algorithm improves bandwidth by exploiting signal properties such as presence of $\mathrm{CP}$ or autocorrelation characteristics in multicarrier signal [2], [3].

The design of time domain equalizer (TDE) extensively studied in the literature [2],[3],[4],[5],[7,18]. Most supervised methods originate from (Falconer and Magee) [5], they proposed a minimum mean-square (MMSE) method for channel shortening, aiming at reducing the complexity in maximum likelihood sequence estimation which has exponentially growth with respect to channel length. Melsa, Younce and Rohrs [4], proposed the maximum shortening SNR, that attempt to minimize the energy of the outside window of interest while keeping the inside energy fixed. Aldhahil and Cioff [8] proposed maximum geometric SNR approach that incorporate the optimization of achievable bit rate into TDE design, in fact all these approaches utilizes some of constraints to insure performance. In contrast to these supervised approaches, Blind methods have been proposed by various scholars recently. Classical multicarrier equalization by restoration of redundancy (MERRY) have been studied in [9]. In [10], [11] fast converging recursive implementation and generalized MERRY have been proposed as improved versions. Sum squared autocorrelation minimization (SAM) proposed in [13], it exploit the second order statistics (SOS) by minimizing the sum of squared autocorrelation of the combined channel equalizer impulse response outside the desired length. In fact the whiteness of the signal and wide sense stationarity assumptions are taken into account, the cost function is defined and optimized using stochastic gradient descent algorithm. Several improvement versions of SAM algorithm that focuses to reduce complexity have been proposed, such as SLAM in [12], GLHSAM in [16]. Another famous approach for blind equalization is carrier nulling algorithm (CNA) initially proposed by De courville et al [19], then improved through lag hopping by Romano and Barbarossa in [17]. The approach utilizes the presence of null carrier in multicarrier transmitter structure, it is well known that null carriers contain zero energy; hence by minimizing the energy falling in these null carriers an adaptive procedure is developed with low complexity. In [20], an improved version termed "maximization of useful-to-null subcarrier energy ratio" was proposed, but the approach has high computation complexity using power iteration method. Considering all these approaches we can see that they rely on redundancies present on the multicarrier structure to find time domain equalizer without considering the effects of equalizer response on useful carriers. This in turn must give inefficient shortened channel which lead to higher bit error rate. 
In this paper we propose the use of constant envelops of the signal at the receiver and derive the Time domain approach for blind equalization. Also we utilizer decision directed cost function to implement multicarrier blind equalization. These two approaches are being compared with the benchmark approaches.

The paper is organized as follows. Section 2 gives the MCSIMO data model, section 3 and 4 present the proposed TDE update criterions and adaptive implementation, section 5 numerical data and simulation is provided and finally section 6 concludes while section 7 provides references.

\section{MULTICARRIER DATA MODEL}

In multicarrier modulation subcarriers are overlapped closely together while the orthogonality is maintained. The procedure is done by mapping the data bits onto complex valued symbols from a given constellation (QPSK, QAM etc.) and applying an inverse discrete Fourier transform using (IFFT) to transform them into time domain sequences. Baseband equivalent MC system model is depicted in figure 1 (next page). $\mathrm{N}$ parallel data sequences denoted by $a_{k, n}$ are grouped into a block $a_{k}$, where $n \in\{1,2, \ldots, N\}$ subcarriers number, $k$ is a block index. The $k^{\text {th }}$ block modulates the different subcarriers using IFFT operation, resulting to a vector of time domain sample denoted by $\mathrm{x}_{k}=\left[x_{k, 1} x_{k, 2}, \ldots x_{k, N}\right]^{T} \quad$ and expressed by $\mathrm{x}_{k}=F^{H} a_{k} \quad$ Where $\mathrm{F}$ is the FFT matrix, $\{F\}_{k, l}=(1 / \sqrt{N}) e^{-j(2 \pi k l) / N}$ and $k, l \in\{0,1, \ldots, N-1\}$ .The last $v$ samples called cyclic prefix (CP) are copied and prepended at the beginning of the block $\mathrm{X}_{k}$ to form the $k^{\text {th }}$ block of length $M=N+v$, given by

$$
\begin{aligned}
& \tilde{\mathrm{x}}_{k}=\left[\tilde{x}_{k M+1}, \tilde{x}_{k M+2}, \ldots \tilde{x}_{k M+m}\right]^{T}= \\
& {\left[x_{k N-P+1}, x_{k N-P+2, . .} x_{k N}, x_{k N+1}, x_{k N+2}, \ldots x_{k N}\right]^{T}}
\end{aligned}
$$

this will result to orthogonality of the subcarriers. Data are transmitted though several channels deployed (through oversampling or multiple antennas) after parallel to serial operation (P/S). Denoting the finite impulse response channel (FIR) of length $L_{h}$ by $h_{1}, h_{2}, \ldots h_{S}$. Assuming transmitter and receiver are well synchronized, the received data at $s$ branch $(s \in\{1,2, \ldots S\})$ is given by

$$
\tilde{r}_{s, k M+m}=\sum_{l=1}^{L_{h}} h_{s, l} \tilde{x}_{k M+m-l}+\tilde{b}_{s, k M+m}
$$

Where: $\tilde{b}_{s, k M+m}$ is the noise sample, assumed to be white i.i.d and $m \in[1,2 \ldots M]$ is the sample index within the block. When $v \leq L_{h}$ then time domain equalizer (TDE) is needed before the FFT operation. For SIMO transceivers, channel shortening is performed by S TDE's $\left[w_{1}, w_{2} \ldots w_{S}\right]$ of length $L_{w}$, whose output is given by

$$
\tilde{y}_{s, k M+m}=\sum_{l=1}^{L w} w_{s, l} \tilde{r}_{s, k M+m-l}=W_{s}^{T} \tilde{\mathbf{r}}_{k M+m}
$$

Where

$W_{s}=\left[w_{s, 1} w_{s, 2 \ldots} w_{s, L w}\right]^{T}, \tilde{\mathbf{r}}_{s, k M+m}=\left[\tilde{r}_{s, k M+m-1 \ldots,} \tilde{r}_{s, k M+m-L w}\right]^{T}$ After adding all $\mathrm{S}$ time domain equalizer (TDE), the outputs are obtained as:

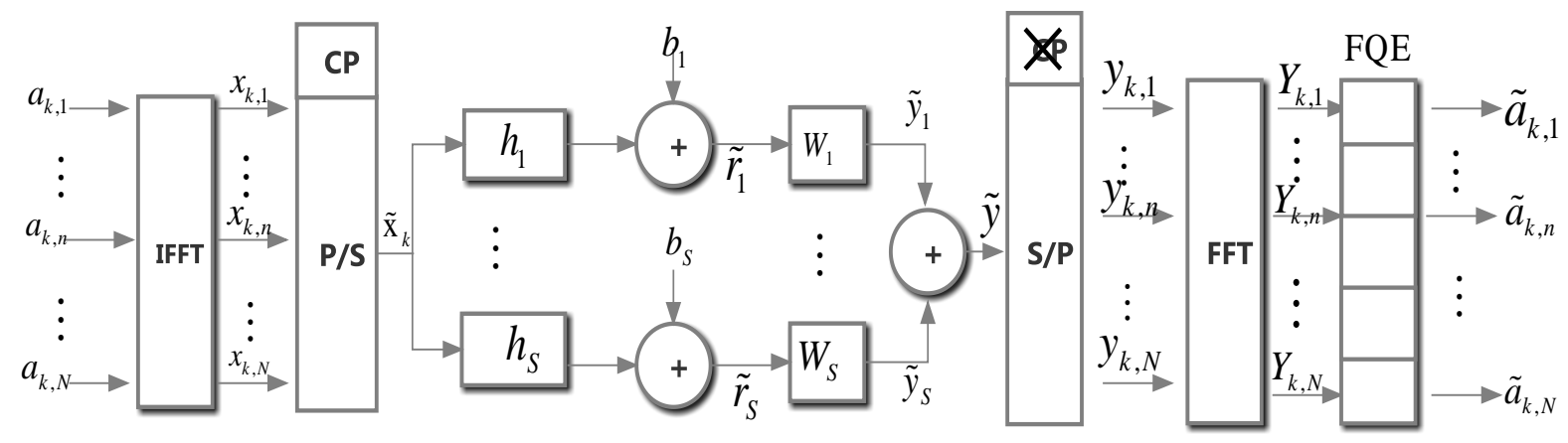

Figure 1, MC-SIMO baseband equivalent system model.

$$
\begin{aligned}
\tilde{y}_{k M+m} & =\sum_{s=1}^{S} \tilde{y}_{s, k M+m} \\
& =\sum_{s=1}^{S} c_{l} \tilde{x}_{k M+m-l}+\sum_{s=1}^{S} \sum_{l=1}^{L w} w_{s, l} \tilde{b}_{s, k M+m-l}
\end{aligned}
$$

Where the effective equivalent channel of length $L_{c}+1$ is:

At the receiver, the effective equivalent channel output is $\mathrm{S} / \mathrm{P}$ converted, M parallel sub-stream are formed, that is $k^{\text {th }}$ block

$\tilde{y}_{k}=\left[\tilde{\mathrm{y}}_{\mathrm{k}, 1} \tilde{\mathrm{y}}_{\mathrm{k}, 2} \ldots, \tilde{\mathrm{y}}_{\mathrm{k}, \mathrm{M}}\right]^{\mathrm{T}}$, the cyclic prefix is removed from the received block $\tilde{y}$ to obtain the $k^{\text {th }}$ received block vector: 


$$
\begin{aligned}
y_{k} & =\left[y_{k, 1} y_{k, 2} \ldots, y_{k, N}\right]^{T} \\
& =\left[\tilde{y}_{k M+P+1} \tilde{y}_{k M+P+2} \ldots, \tilde{y}_{(k+1) M}\right]^{T}
\end{aligned}
$$

At this point serial streams are converted back to parallel. The very important parameter at this stage is synchronization delay $\delta$ that determines where the receiver should break the inputs samples into blocks.

$$
\mathrm{y}(k)=\left[\mathrm{y}_{k M+v+1+\delta}, \mathrm{y}_{k M+v+2+\delta}, \ldots, \mathrm{y}_{k M+v+N+\delta}\right]^{T}
$$

Thereafter, each block is passed through Fast Fourier Transform (FFT) as:

$$
Y(\mathrm{k})=\mathbf{F}\{\mathbf{y}(\mathrm{k})\}
$$

The output of FFT for each tone is equalized by one tap frequency domain equalizer (FEQ) to scale and insure proper orientation of the symbol.

$$
a_{k, i}=Y(\mathrm{k}) \cdot \mathrm{FEQ}_{i}
$$

Where $\mathrm{k}$ and $i$ denote the $k^{\text {th }}$ block and $i^{\text {th }}$ sample respectively. The effective channel is expressed as the sum of convolution of each channel with its respective time domain equalizer (TDE)That is:

$$
c=c_{1}+c_{2}
$$

$$
\text { Where } \quad c_{1}=h_{1} * w_{1} \text { and } c_{2}=h_{2} * w_{2}
$$

\section{CONSTANT MODULUS ALGORITHM FOR A TDE}

Constant modulus approach has been used widely in single carrier systems; here we develop an adaptive criterion for multicarrier systems. Consider the cost function defined at the output of the FFT and frequency domain equalization FQE:

$$
J_{C M}=\sum_{i=1}^{N} E\left[\left(\left|\tilde{a}_{k, i}\right|^{2}-R_{i}\right)^{2}\right]
$$

Where $a_{k, i}, R_{i}$ denotes the particular tone and statistics of the transmitted sequences. Using the instantaneous stochastic gradient descent optimization procedure, we have:

$$
\frac{\partial J_{C M}}{\partial w}=\sum_{i=1}^{N} \frac{\partial\left|e_{i}\right|^{2}}{\partial w}=\sum_{i=1}^{N}\left(\nabla_{w} e_{i}\right) e_{i}^{*}+e_{i}\left(\nabla_{w} e_{i}^{*}\right)
$$

Where $e_{\mathrm{i}}=\left(\left|\tilde{a}_{k, i}\right|^{2}-R_{i}\right)$. Recalling that the gradient with respect to complex vector is given by:

$$
\frac{\partial \tilde{a}_{k, i} \tilde{a}_{k, i}^{*}}{\partial w_{l}}=0.5\left(\frac{\partial \tilde{a}_{k, i} \tilde{a}_{k, i}^{*}}{\partial w_{l, R}}+j \frac{\partial \tilde{a}_{k, i} \tilde{a}_{k, i}^{*}}{\partial w_{l, I}}\right)
$$

Where $\mathrm{R}$, I and * refers to real, imaginary and complex conjugate respectively. It can be shown that:

$$
\frac{\partial \tilde{a}_{k, i}}{\partial w}=0
$$

$$
\frac{\partial \tilde{a}_{k, i}^{*}}{\partial w}=2 F E Q^{*} \sum_{l=1}^{N} f_{l, i}^{*} \tilde{r}_{1}^{*}(\mathrm{Mk}+1)
$$

After further manipulations of (12) we arrive at:

$$
\begin{aligned}
& \frac{\partial J_{M C C M A}}{\partial w_{1}}=4 \sum_{i=1}^{N} e_{i}\left(a_{k, i}\left[F E Q_{i}^{*} \sum_{l=1}^{N} f_{l, i}^{*} \tilde{r}_{1}^{*}(\mathrm{Mk}+1)\right]\right) \\
& \frac{\partial J_{M C C M A}}{\partial w_{2}}=4 \sum_{i=1}^{N} e_{i}\left(a_{k, i}\left[F E Q_{i}^{*} \sum_{l=1}^{N} f_{l, i}^{*} \tilde{r}_{2}^{*}(\mathrm{Mk}+1)\right]\right)
\end{aligned}
$$

Where

$$
\tilde{r}_{1,2}(M k+l)=r_{1,2}\left(M k+l+1:-1: M k+l-L_{w}+1\right)^{T}
$$

The two time domain equalizers (TDE) can be updated by the procedure below, noting that $\mu$ is the step size.

$$
\begin{aligned}
& W_{1, k+1}=W_{1, k}-\mu \nabla_{W_{1}} J_{M C C M A} \\
& W_{2, k+1}=W_{2, k}-\mu \nabla_{W_{2}} J_{M C C M A}
\end{aligned}
$$

\section{DECISION-DIRECTED ALGORITHM (DDA) FOR TDE}

The Decision-directed algorithm selects the nearest symbol in the constellation relatively to the current received symbol estimate and the equalizer is adapted by minimizing the mean square error (MMSE) of the distance between them. For single carrier the cost function is defined as: $J_{D D A}=E\left[\left|Q\left[a_{k}\right]-a_{k}\right|^{2}\right]$

We define the multicarrier cost function as:

$$
J_{M C D D A}=\sum_{i=1}^{N} J_{D D A}=\sum_{i=1}^{N}\left|e_{i}\right|^{2}=\sum_{i=1}^{N} e_{i} e_{i}^{*}
$$

Where $e=Q\left[a_{k}\right]-a_{k}$ and we have considered instantaneous cost function, thus expectation operator is removed. Taking the stochastic gradient descent of the cost function with respect to equalizer vectors as:

$$
\frac{\partial J_{M C D D A}}{\partial w}=\sum_{i=1}^{N} \frac{\partial\left|e_{i}\right|^{2}}{\partial w}=\sum_{i=1}^{N}\left(\nabla_{w} e_{i}\right) e_{i}^{*}+e_{i}\left(\nabla_{w} e_{i}^{*}\right)
$$

The gradient of the output of quantizer is zero, since it is constant. After modest algebra and keeping in mind equations (14) and (15) we realize that:

$$
\begin{aligned}
& \frac{\partial J_{M C D D A}}{\partial w_{1}}=-2 \sum_{i=1}^{N} e_{i}\left(\left[F E Q_{i}^{*} \sum_{l=1}^{N} f_{l, i}^{*} \tilde{r}_{1}^{*}(\mathrm{Mk}+1)\right]\right) \\
& \frac{\partial J_{M C D D A}}{\partial w_{2}}=-2 \sum_{i=1}^{N} e_{i}\left(\left[F E Q_{i}^{*} \sum_{l=1}^{N} f_{l, i}^{*} \tilde{r}_{2}^{*}(\mathrm{Mk}+1)\right]\right)
\end{aligned}
$$

The two time domain equalizers (TDE) can be updated by the procedure below, noting that $\mu$ is the step size.

$$
W_{1, k+1}=W_{1, k}-\mu \nabla_{W_{1}} J_{M C D D A}
$$




$$
W_{2, k+1}=W_{2, k}-\mu \nabla_{W_{2}} J_{M C D D A}
$$

\section{SIMULATIONS AND DISCUSION}

In this section we perform numerical simulation for the adaptive blind channel shortener derived. The simulation parameters used are typical to that of wireless LAN standard IEEE 802.11a: 4 QAM (QPSK) signaling was used, on which we generated 10,000 number of FFT blocks each block have a size of 64 (i.e. 64 FFTSIZE) and cyclic prefix of length 16.

The Rayleigh channel model with exponentially distribution profile was generated with 32 taps. Two equalizer (system with two antennas $\mathrm{S}=2$ ) of length 48 was used. MERRY, CNA and multicarrier training algorithms (MCTA) were used for comparison purpose. Step size parameters used for the algorithms are shown in Table 1

Table 1. Step size used in algorithms

\begin{tabular}{lll}
\hline Algorithm & FQE $(\mu)$ & $\operatorname{TDE}(\mu)$ \\
\hline MCT & 0.005 & 0.00002 \\
MCDD & 0.005 & 0.000003 \\
MCCM & 0.005 & 0.000005 \\
MERRY & 0.005 & 0.0003 \\
CNA & 0.005 & 0.000003 \\
\hline
\end{tabular}

Two simulations where performed. One is when the system has null carriers and the other when the system has no null carriers. Fig. 2 below shows channel response before equalization (shortening) and Fig. 3 show after equalization with MCTA (multicarrier trained algorithm), only taps energy left are within CP length.

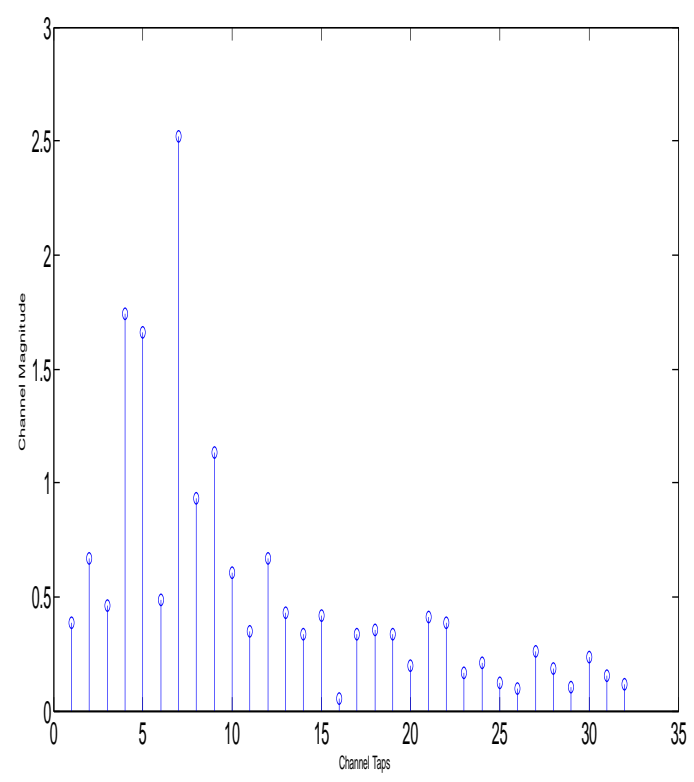

Figure 2: Magnitude of Rayleigh Fading channel

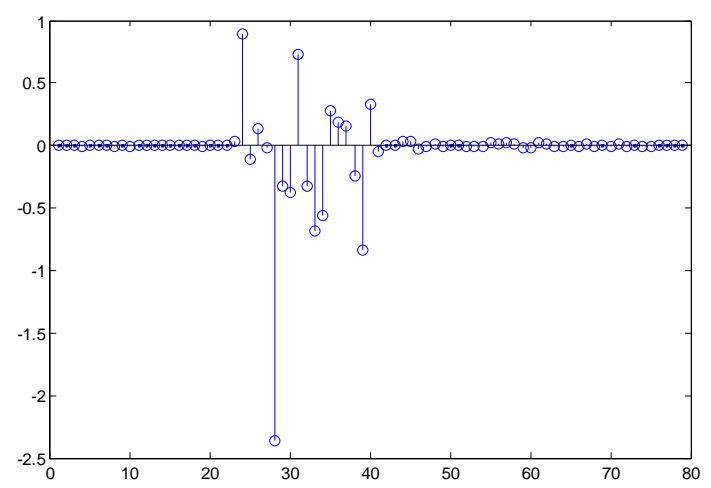

Figure 3: Shortened channel impulse response using Multicarrier Training algorithm.

Fig 4. Show the performance of the four algorithms in terms of BER (Bit error rate) as a function of signal to noise ratio (SNR), when the null carriers are not used. It can be seen that the proposed methods for TDE design outperform the famous MERRY algorithm. The MCDDA had reduced performance compared to MCTA as expected, but it is spectral efficiency compared to MCTA since no training is required during equalizer adaptation. MCCMA performs well compared to MERRY algorithm as it adapts the equalizer several times per block. In fact the MERRY algorithm adapts only once per OFDM block hence it needs more data to offer better Performance. MCCMA cost function is fourth order polynomial hence it suffers from multimodality cost surface, therefore it must have poor convergence compared to MCDDA and MCTA.

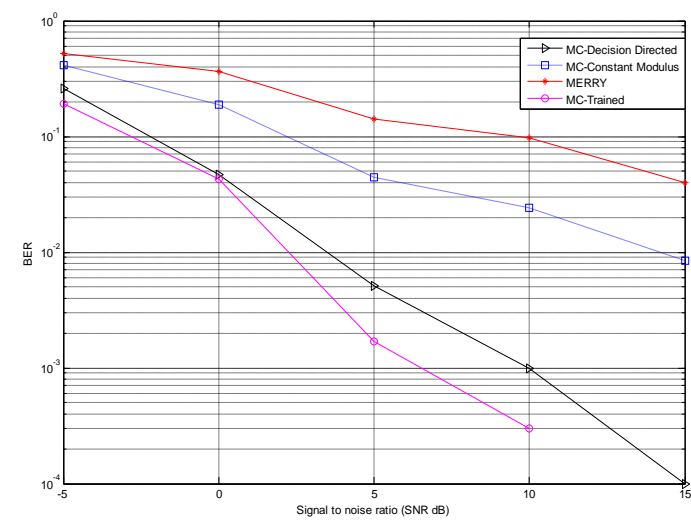

Figure 4. BER comparison for four algorithms without null carries

In case we introduce some carriers with zero energy the BER against SNR is depicted on the Fig. 5 below. 12 null carriers were used on which 6 were inserted at the beginning of the FFT block and 6 where inserted at the end of the FFT block. The BER performance for these algorithms does not change much when more number of FFT block is used to adapt the equalizer, otherwise the performance of MCDDA and MCCMA would have to degrade seriously since they have no information on the presence of zero energy in some carriers as MCTA do. CNA (carrier null algorithm) is also compared with others blind algorithm as depicted in the figure 5 . 


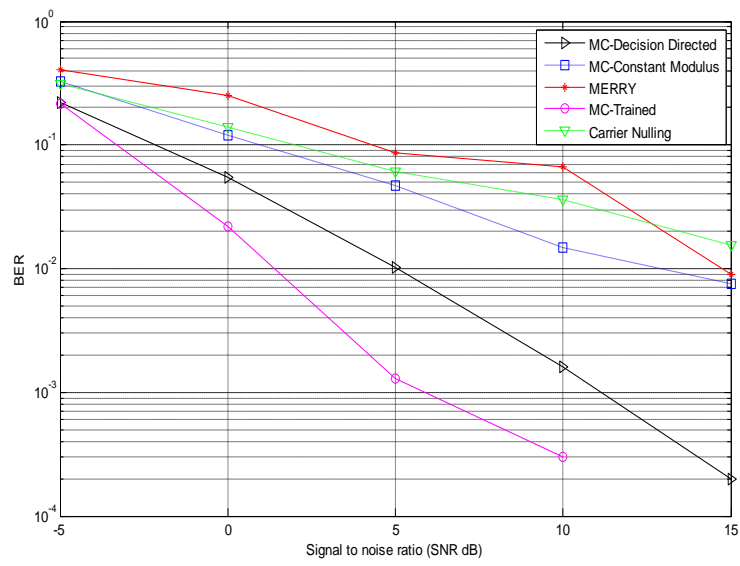

Figure 5: BER comparison for five algorithms with null carries

\section{CONCLUSION}

In this article we have introduces the BER performance of two bind channel shortening algorithms for multicarrier systems. When the delay spread of the channel is greater than $\mathrm{CP}$, time domain equalizer is important for shortening the channel impulse response. We have compared the performance of the derived algorithms relative to supervised and other unsupervised methods, it have been observed that algorithms that depends on redundancies requires more data for updating the equalizer so as to have better convergence, incorporating fractionally equalization structure has improved the performance of the constant modulus algorithm in multicarrier system (MCCMA). For this perspective we have seen that the proposed methods outperform other blind methods. More analysis in terms of computation complexity, presence of colored noise and other interferes is currently being investigated. Incorporating channel coding methods and MIMO system might further improves the proposed blind methods.

\section{REFERENCES}

[1] Z. wang and G.B Giannakis, "Wireless Multicarrier Communication" IEEE Signal Processing Mag., vol 17 no. 3, pp 29-48, may 2000.

[2] J. M. Walsh, R. K. Martin, and C. R. Johnson, Jr. "Convergence and performance issues for autocorrelation based adaptive channel shortening," in Asilomar Conference on Signals, Systems, and Computers, Nov, 2006.

[3] Balakrishnan J., Martin, R.K., and Johnson, C.R.: "Blind, adaptive channel shortening by sum-squared autocorrelation minimization (SAM)", IEEE Trans. Signal Process., 2003, 51, (12), pp. 3086-3093

[4] P. J. W. Melsa, R. C. Younce, and C. E. Rohrs, "Impulse response shortening for discrete multitone transceivers," IEEE Trans. Commun., vol. 44, pp. 16621672, Dec. 1996

[5] D. D. Falconer and F. R. Magee, "Adaptive Channel Memory Truncation for Maximum Likelihood Sequence Estimation," Bell Sys. Tech. Journal, pp. 1541-1562, Nov. 1973.

[6] R. K. Martin, "Blind, adaptive equalization for multicarrier receivers," Ph. D. Thesis, Cornell University, US 2004.
[7] G. Arslan, M. Ding, B. Lu, Z. Shen, and B. L. Evans, "TEQ Design Toolbox. Univ.Texas, Austin, TX. [Online.] Available:http://www.ece.utexas.edu/bevans/projects/ads 1/dmtteq/dmtteq.html,"

[8] N. Al-Dhahir and J. Cioffi,"The combination of Finite Length Geometric Equalization and Bandwidth Optimization for Multicarrier Transceivers" in International conf. on Acoustic, Speech and Signal Processing, May 1995 pp. 1201-1204.

[9] R.k Martin, J Balakrishnan, W.A Sethares, and CR Johnson, Jr "A blind adaptive TQE for Multicarrier Systems", IEEE Trans. Commn. Vol 49, no 11, Nov 2002 pp. 341-343.

[10] R.k Martin, "Fast-Converging Blind Adaptive ChannelShortening and Frequency- Domain Equalization" IEEE Trans.on Signal Processing Vol. 55, No 1, Jan 2007, pp 102-110

[11] R. K. Martin, J. M. Walsh, and C. R. Johnson, Jr.,"Low complexity MIMO blind, adaptive channel shortening," IEEE Trans. Signal Process., vol. 53, no. 4, pp. 1324 1334, Apr. 2005

[12] R. Nawaz and J.A. Chambers, "Blind Adaptive Channel Shortening by Single Lag Autocorrelation Minimization (SLAM)," Electronics Letters, vol. 40, pp. 1609-1611, December 2004.

[13] J. Balakrishnan, R. K. Martin, and C. R. Johnson, Jr., "Blind, adaptive channel shortening by sum-squared auto-correlation minimization (SAM),'IEEE Trans. Signal Process. vol. 51, no. 12, pp. 3086-3093,Dec. 2003

[14] M.Yamin and R.Nawaz, "A Blind adaptive channel shortening algorithm using any lag auto correlation minimization" Emerging Technologies (ICET) Sept. 2011.

[15] M.Grira, J.A Chambers "A blind lag-hopping adaptive channel shortening algorithm based upon sum-squared autocorrelation minimization (LHSAM)" Acoustic speech and signal processing 2008 pp. 3569-3572.

[16] K. Maatong, J.A Chambers "Blind adaptive channel shortening with a Generalized Lag hopping which employs sum-squared autocorrelation minimization GLHSAM" ICSNC 2008, pp. 75-78

[17] F. Romano and S. Barbarossa, "Non-data aided adaptive channel shortening for efficient multi-carrier systems," in Proc. of Intl. Conf. on Acoustics, Speech and Signal Proc., pp. 233-236 Apr. 2003.

[18] T. B. Jabeur, K. Abed-Meraim, and H. Boujemaa, "Blind channel shortening in OFDM systems using nulltones and cyclic prefix," in Proc. of ICASSP, pp. 3041-3044, 2008.

[19] M. de Courville, P. Duhamel, P. Madec, and J. Palicot,"Blind equalization of OFDM systems based on the minimization of a quadratic criterion,"inProc. IEEE Int. Conf. Communications, Dallas, TX, Jun. 1996, pp. 1318-1321.

[20] E.B. Salem, H. Besbes "Maximization of useful-to-null subcarrier energy ratio for blind multicarrier SIMO channel shortening" 7th European Signal Processing Conference (EUSIPCO 2009) 